\title{
Source-assisting Strategy for Distributed Space-time Block Codes
}

\author{
Gbenga Owojaiye ${ }^{1}$, Fabien Delestre ${ }^{2}$, Yichuang Sun $^{3}$ \\ School of Engineering and Technology \\ University of Hertfordshire \\ Hatfield, AL10 9AB United Kingdom \\ $\left\{{ }^{1}\right.$ g.owojaiye, ${ }^{2}$ F.Delestre, ${ }^{3}$ Y.Sun $\}$ @herts.ac.uk
}

\begin{abstract}
Most of the previous works on distributed space-time coding assume that there is no direct link between the source and the destination, which means that the source is not actively involved in cooperation. In order to present the benefits that can be extracted when the source is actively involved in cooperation, we propose a source-assisting (SA) strategy for distributed orthogonal and quasi-orthogonal space-time block coded cooperative multi-hop networks. We adopt a two time-slot protocol using the decode-and-forward model, where in the first time slot, the source transmits while the relay and destination nodes all receive, and in the second time slot, the source and relay nodes transmit and the destination node receives. Numerical and simulation results show that our SA strategy exhibits improved diversity performance compared to the conventional distributed orthogonal and quasi-orthogonal designs, at the expense of slightly increased complexity of the ML detector. An explanation on the modified structure of the ML detector is provided.
\end{abstract}

Keywords-- Distributed space-time codes, source-assisting distributed orthogonal space-time block codes, source-assisting distributed quasi-orthogonal space-time block codes

\section{INTRODUCTION}

Increasing interest in Adhoc and wireless sensor network technologies has led to extensive research aimed at extending the benefits of spatial diversity in multiple antenna systems, to the single antenna nodes of multi-hop networks. This paradigm is referred to as 'cooperative diversity' where the nodes that constitute multi-hop networks are made to share their single antennas thereby creating a virtual multiple antenna array. Different strategies have been employed to implement cooperative diversity in multi-hop networks of which repetition-based coding [1] [2] and distributed space time block codes (DSTBC) [3] [4] appear to be the most popular. However, the sub-optimum bandwidth performance of repetition-based coding has instigated further development of DSTBC algorithms.

The idea of DSTBC is based on employing space time block codes (STBC) originally designed for multiple antenna systems, in a distributed fashion for multi-hop networks. This idea was first introduced by Laneman et al in [5] for two major models; the decode-and-forward (DF) model where the intermediate node decodes the information signal received from the source node before retransmission and the amplifyand-forward (AF) model where the intermediate node simply amplifies the received signal before retransmission. These two major models have been extensively employed in the design of relaying protocols for cooperative networks.

As space-time codes designed for multiple antenna systems become readily extendable to the distributed nature of multi-hop networks, many researchers exploit this idea by extending the STBC designs to single antenna multi-hop networks and investigate the performance. In [6] for example, the authors propose distributed GABBA codes using the AF model where each relay transmits a scaled version of the linear combination of its received symbols, similarly in [7] the authors applied linear dispersion codes in AF cooperative networks and studied the achievable diversity order. In [8] Rajan et al investigated the use of generalized coordinate interleaved orthogonal designs and unitary weight single symbol decodable codes originally developed for multiple antenna systems, their results show satisfactory performance. In [9] the author focused on the design of distributed differential space-time codes for a two-way relay network where the terminals have no knowledge of the fading channels. Similar to our work, in [10] the authors propose the use of orthogonal space time block codes in wireless relay networks, in their work they compare the performance of DSTBC with selection DF models.

In all the aforementioned works, the source node is not involved in cooperation, thus there is no direct link between the source and the destination. However considering that additional benefits can be exploited in terms of diversity and error performance when the source is actively involved in cooperation, we propose a source-assisting (SA) strategy for DF cooperative networks by employing orthogonal and quasiorthogonal STBC originally designed for multiple antenna systems. From this, we model the source-assisting distributed orthogonal STBC (SA-DOSTBC) and the source-assisting distributed quasi-orthogonal STBC (SA-DQOSTBC). Our approach allows us to directly apply the SA strategy on existing STBC used in [11] and [12] originally developed for multiple antenna systems. We first show how to apply the SA strategy on a single relay network $R_{n}=1$, we then implement for networks where the number of relay nodes $R_{n}=3$ are involved in cooperation. For both cases, we adopt a time sharing approach where we allocate separate time slots to inter-node transmissions, the time slot approach is clarified 
subsequently. We then present the benefits that can be achieved when the source is actively involved in cooperation.

The rest of the paper is organized as follows: In Section II, the considered cooperative network model is presented and the proposed SA strategy is introduced. Design criteria for the SA-DOSTBC and SA-DQOSTBC are given in Section III, while the benefits extracted from the SA strategy are discussed in Section IV. In Section V, simulation results are provided and Section VI concludes the paper.

\section{SYSTEM MODEL}

Consider a multi-hop network with a source node $S$, intermediate relay nodes $R_{n}=R_{1}, \ldots, R_{N}$ and destination node $D$ as shown in Fig. 1, all nodes in the network are each assumed to have a single antenna. The model adopts a time sharing approach such that the transmission interval between $S$ and $D$ is sub-divided into time slots $t_{1}$ and $t_{2}$, with synchronous transmission between transmitting nodes per time slot. In the first time slot $t_{1}, S$ transmits $N$ information symbols from an $m=\log _{2} M$ signal constellation. We assume that at $t_{1}, R_{n}$ receives the signal vector transmitted from $S$ while $D$ is set to idle mode such that it does not receive the transmission from $S$, later a case where $D$ is set to receiving mode at $t_{1}$ will be presented. Let $s=\left[s_{1}, \ldots, S_{N}\right]$ denote the symbol vector transmitted from $S$ at $t_{1}$, we model the source-relay channel $h_{S R}$ as a Rayleigh flat fading channel where the coherence time of $h_{S R}$ is larger than $N$. Then the received signal vector at the $n_{t h}$ relay node assuming perfect CSI with the DF model implemented is given as:

$$
r_{n}=h_{S R} s_{n}+n_{n}
$$

where $n_{n}=\left[n_{1}, \ldots, n_{N}\right]$ is the additive white Gaussian noise. To preserve the relay simplicity, we enable symbol-by-symbol detection such that $R_{n}$ decodes and provides estimates $\tilde{s}_{n}=\left[\tilde{s}_{1}, \ldots, \tilde{s}_{N}\right]$.

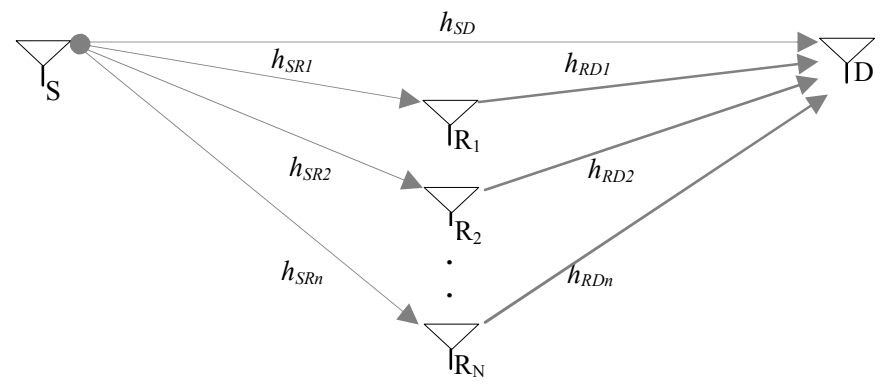

Fig. 1. Cooperative Network Model

In order to construct a DSTBC at $t_{2}$, the source transmits:

$$
x_{S}=\left[s_{1}, \ldots, s_{N}\right]
$$

and the $n_{t h}$ relay node transmits:

$$
x_{n}=\left[\tilde{s}_{1}, \ldots, \tilde{s}_{N}\right]
$$

Such that the transmitted signal by the $n_{t h}$ relay node is designed to be a linear function of its received signal. The signal received at $D$ assuming perfect CSI is:

$$
r_{d}=h_{S D} x_{s}+h_{R D} x_{n}+n_{d}
$$

Where $n_{d}=\left[n_{d, 1}, \ldots, n_{d, N}\right]$ is the additive white Gaussian noise assumed to be i.i.d random variables. We denote $h_{n}=\left\{h_{S D}, h_{R D}\right\}$ and $C_{n}=\left\{S, R_{n}\right\}$ as the set of channel coefficients and cooperating nodes respectively at $t_{2}$ where $h_{S D}$ is the channel between $S$ and $D$, and $h_{R D}$ is the channel between $R$ and $D$. We leverage perfect transmit synchronization between all $C_{n}$, and model $h_{n}$ as i.i.d Rayleigh flat fading channels with coherence time larger than $N$. It is now assumed that for all transmissions at $t_{2}$, only the deterministic set of nodes $C_{n}$ are involved in cooperation, such that the nodes transmit in $T \geq C_{n}$ consecutive symbol intervals.

For the design of complex valued DOSTBC, we follow the design of relative OSTBC such that the DOSTBC design problem is like an OSTBC design problem, where the entries of matrix $G$ can contain either the information bearing symbols $s_{1}, \ldots, s_{N}$ or their conjugates $s_{1}^{*}, \ldots, s_{N}^{*}$ on its $i_{t h}$ column.

\section{ORTHOGONAL AND QUASI-ORTHOGONAL DESIGNS}

\section{A. Distributed Orthogonal Space-time Block Codes}

To leverage the code design of matrix $G$, we conclude hypothetically that OSTBC that can be designed such that their columns only contain $s_{1}, \ldots, S_{N}$ or their conjugates $s_{1}^{*}, \ldots, s_{N}^{*}$ can be applied in a distributed fashion. We show the application of our SA strategy for a single relay network $R_{n}=1$, where the Alamouti design [11] is applied to suite the requirements of matrix $G$ without disrupting its structure. Reiterating the transmission pattern for $t_{1}$ and $t_{2}$ as in Section II, we implement the DOSTBC design only at $t_{2}$ such that the source now transmits:

$$
x_{S}=\left[s_{1},-s_{2}^{*}\right]
$$

and the relay node transmits:

$$
x_{n}=\left[\tilde{s}_{2}, \tilde{s}_{1}^{*}\right]
$$

for each $i_{t h}$ transmission interval where $i=1,2$ for the Alamouti design. Applying the channel characteristics and 
CSI as in Section II, the $i_{t h}$ signal received at $D$ at $t_{2}$ becomes:

$$
\begin{gathered}
r_{d, 2}=\left[r_{i+1}, r_{i+2}\right] \\
r_{i+1}=h_{S D} s_{1}+h_{R D} \tilde{s}_{2}+n_{i+1} \\
r_{i+2}=-h_{S D} s_{2}{ }^{*}+h_{R D} \tilde{s}_{1}{ }^{*}+n_{i+2}
\end{gathered}
$$

From (8) above it is obvious that the conjugates of the transmitted symbols are only received in the second transmission interval $i+2$, thus the matrix $G$ at the receiver is of the form: $\left[\begin{array}{cc}s_{1} & \tilde{s}_{2} \\ -S_{2}^{*} & \tilde{S}_{1}^{*}\end{array}\right]$ which meets the defined structure for complex orthogonal designs even when the source is actively involved in cooperation. Thus we are able to demonstrate that with regards to $C_{n}$, a diversity order of $d=\min \left\{C_{n}, T\right\}$ can be achieved when all $R_{n}$ correctly decode their received information symbols at $t_{1}$. Also, depending on the design of the code matrix, the proposed SA strategy can achieve improved diversity and low complexity ML decoding, this aspect is discussed later.

\section{B. Distributed Quasi-orthogonal Space-time Block Codes}

For design of the SA-DQOSTBC, we extend our analysis to a cooperative network with $R_{n}=3$ relay nodes such that $C_{n}=\left\{S, R_{n}\right\}$. In the first time slot $t 1$, we reiterate the SISO transmission process between $S$ and all $R_{n}$ as in previous sections, the only difference being we increase the symbol length of the information signal $s_{i}$ for $i=1, \ldots, 4$. In the second time slot $t_{2}$, we leverage the DQOSTBC design using the QOSTBC design proposed in [12] by Jafarkhani et al in order to generate the structure of matrix $G$ at the receiver, different from [10] we impose our SA strategy such that the codewords are optimally distributed between $S$ and all $R_{n}$. We implement the SA-DQOSTBC design only at $t_{2}$ such that the source now transmits:

$$
x_{s}=\left[s_{1},-s_{2}^{*},-s_{3}^{*}, s_{4}\right]
$$

and the relay nodes transmit:

$$
\begin{aligned}
& x_{n 1}=\left[\tilde{s}_{2}, \tilde{s}_{1}^{*},-\tilde{s}_{4}^{*},-\tilde{s}_{3}\right] \\
& x_{n 2}=\left[\tilde{s}_{3},-\tilde{s}_{4}^{*}, \tilde{s}_{1}^{*},-\tilde{s}_{2}\right] \\
& x_{n 3}=\left[\tilde{s}_{4}, \tilde{s}_{3}^{*}, \tilde{s}_{2}^{*}, \tilde{s}_{1}\right]
\end{aligned}
$$

From (9) and (10) above we can see that the entries of the $C_{n} \times T$ matrix $G$ received at the destination $D$ only contain complex conjugates on entire columns, which ensures quasi-orthogonality. Thus the resultant signal received at $D$ at $t_{2}$ is: $r_{d, 2}=\left[r_{i+3}, r_{i+4}, r_{i+5}, r_{i+6}\right]$

$r_{i+3}=h_{S D} s_{1}+h_{R_{1} D} \tilde{s}_{2}+h_{R_{2} D} \tilde{s}_{3}+h_{R_{3} D} \tilde{s}_{4}+n_{i+3}$
$r_{i+4}=-h_{S D} s_{2}^{*}+h_{R_{1} D} \tilde{s}_{1}^{*}-h_{R_{2} D} \tilde{s}_{4}^{*}+h_{R_{3} D} \tilde{s}_{3}^{*}+n_{i+4}$ $r_{i+5}=-h_{S D} s_{3}^{*}-h_{R_{1} D} \tilde{s}_{4}^{*}+h_{R_{2} D} \tilde{s}_{1}^{*}+h_{R_{3} D} \tilde{s}_{2}^{*}+n_{i+5}$ $r_{i+6}=h_{S D} s_{4}-h_{R_{1} D} \tilde{s}_{3}-h_{R_{2} D} \tilde{s}_{2}+h_{R_{3} D} \tilde{s}_{1}+n_{i+6}$

\section{BENEFITS EXTRACTED FROM THE SOURCE-ASSISTING STRATEGY}

In order to present the benefits that can be extracted when the source is actively involved in cooperation, we first provide an explanation on the modified structure of the signal combiner at $D$ for the SA-DOSTBC described in Section IIIA. Conjugating the received signal $r_{i+2}$ in (8), the received signal at $D$ at $t_{2}$ becomes: $r_{d, 2}=\left[r_{i+1}, r_{i+2}^{*}\right]$

$$
\begin{aligned}
& r_{i+1}=h_{S D} s_{1}+h_{R D} s_{2}+n_{i+1} \\
& r_{i+2}^{*}=h_{R D}^{*} s_{1}-h_{S D}^{*} s_{2}+n_{i+2}^{*}
\end{aligned}
$$

Assuming $D$ is switched to receiving mode at $t_{1}$, we introduce the vector $r_{d, 1}=\left[r_{0}, r_{i}\right]$ as the information symbols received at $D$ from $S$ at $t_{1}$ in the $i_{t h}$ transmission interval conditioned on $s_{i}=\left[s_{1}, s_{2}\right]$. Thus the information signals received at $D$ at $t_{1}$ is given by:

$$
\begin{aligned}
& r_{0}=h_{S D_{1}} s_{1}+n_{0} \\
& r_{i}=h_{S D_{1}} s_{2}+n_{i}
\end{aligned}
$$

The equivalent signal received at $D$ with the SA strategy implemented is obtained by combining (12) and (13), and is given in short notation as: $r_{d}=\left[r_{d, 1}, r_{d, 2}\right]=H s+n$ where $H$ denotes the equivalent channel matrix between all $C_{n}$ and $D$, and is given as:

$$
H=\left[\begin{array}{cc}
h_{S D_{1}} & 0 \\
0 & h_{S D_{1}} \\
h_{S D} & h_{R D} \\
h_{R D}^{*} & -h_{S D}^{*}
\end{array}\right]
$$

If the ML detector can perfectly estimate the channel coefficients $h_{S D_{1}}, h_{S D}$ and $h_{R D}$, they can be used as CSI. Let, and $s=\left\{\overline{s_{1}}, \overline{s_{2}}\right\}$ denote the set of all possible transmitted vectors estimated by the ML detector which minimizes the Euclidean distance $J$ from a signal constellation $M$ with equal probabilities, thus $J=\left|r_{d}-H s\right|^{2} \quad$ where $r_{d}=\left[r_{0}, r_{i}, r_{i+1}, r_{i+2}\right]$, substituting for $r_{i+1}$ and $r_{i+2}$ in (12) and $r_{0}$ and $r_{i}$ in (13) we have:

$J=\left|r_{0}-h_{S D_{1}} \bar{S}_{1}\right|^{2}+\left|r_{i}-h_{S D} \bar{S}_{2}\right|^{2}+\left|r_{i+1}-h_{S D} \bar{s}_{1}-h_{R D} \bar{S}_{2}\right|^{2}+\left|r_{i+2}+h_{S D} \bar{s}_{2}^{*}-h_{R D} \bar{S}_{1}^{*}\right|^{2}$ In order to recover estimates $\widehat{S}_{1}$ and $\widehat{S}_{2}$ of the transmitted 
information signal, the combiner combines $r_{0}, r_{i}, r_{i+1}$ and $r_{i+2}$ such that: $\widehat{S}_{1}=h_{S D_{1}}^{-1} r_{0}+h_{S D}^{*} r_{i+1}+h_{R D} r_{i+2}^{*}$

$=\left(1+\left|h_{S D}\right|^{2}+\left|h_{R D}\right|^{2}\right) s_{1}+h_{S D_{1}}^{-1} n_{0}+h_{S D}^{*} n_{i+1}+h_{R D} n_{i+2}^{*}$

$\widehat{s}_{2}=h_{S D_{1}}^{-1} r_{i}+h_{R D}^{*} r_{i+1}-h_{S D} r_{i+2}^{*}$

$$
=\left(1+\left|h_{S D}\right|^{2}+\left|h_{R D}\right|^{2}\right) s_{2}+h_{S D_{1}}^{-1} n_{i}+h_{R D}^{*} n_{i+1}-h_{S D} n_{i+2}^{*}
$$

From (14) above we can see that the two information symbols can be recovered separately indicating low-complexity decoding. Also, by switching $D$ to receiving mode at $t_{1}$, the model exhibits improved diversity performance at the cost of slightly increased complexity of the ML detector, notably the additional power gain and the noise term scaled by a factor $h_{S D_{1}}^{-1}$. This improved diversity performance is shown in the simulation results in Section V. It is obvious that in our explanation of the structure of the ML detector we relax the limiting factor $\tilde{s}_{n} \neq s$ assumed in Section II such that there is no confusion, in other words we assume that the relay node correctly decodes the information symbols received at $t 1$.

Next, to discuss the improved diversity offered by the SADQOSTBC discussed in Section IIIB, we provide an explanation on the modified structure of the signal combiner at $D$. Conjugating the received signal $r_{i+4}$ and $r_{i+5}$ in (11), the received signal at $D$ at $t_{2}$ becomes:

$$
r_{d, 2}=\left[r_{i+3}, r_{i+4}^{*}, r_{i+5}^{*}, r_{i+6}\right]
$$

Assuming $D$ is switched to receiving mode at $t_{1}$, we introduce the vector $r_{d, 1}=\left[r_{0}, r_{i}, r_{i+1}, r_{i+2}\right]$ as the information symbols received at $D$ from $S$ in the $i_{t h}$ transmission interval conditioned on $s_{i}=\left[s_{1}, s_{2}, s_{3}, s_{4}\right]$. Thus the information signals received at $D$ at $t_{1}$ is given by:

$r_{0}=h_{S D_{1}} s_{1}+n_{0}$

$r_{i}=h_{S D_{1}} s_{2}+n_{i}$

$r_{i+1}=h_{S D_{1}} s_{3}+n_{i+1}$

$r_{i+2}=h_{S D_{1}} s_{4}+n_{i+2}$

The equivalent signal received at $D$ with the SA strategy in place is obtained by combining (15) and (16), and is given in short notation as: $r_{d}=\left[r_{d, 1}, r_{d, 2}\right]=H s+n$

where $H$ denotes the equivalent channel matrix between all $C_{n}$ and $D$. In order to recover the transmitted information signals, the combiner obtains a new decision vector $v=\left[v_{1}, v_{2}, v_{3}, v_{4}\right]^{T}$, given by:

$v=H^{\mathrm{H}} r_{d}=H^{\mathrm{H}} H s+H^{\mathrm{H}} n$

$v=M s+H^{\mathrm{H}} n$
Where $M$ is the Gramian matrix which dictates the complexity of the decoding algorithm and is given as:

$M=H^{\mathrm{H}} H=H H^{\mathrm{H}}=h^{2}\left[\begin{array}{cccc}1 & 0 & 0 & \beta \\ 0 & 1 & -\beta & 0 \\ 0 & -\beta & 1 & 0 \\ \beta & 0 & 0 & 1\end{array}\right]$

$h^{2}=\left(\left|h_{S D_{1}}\right|^{2}+\left|h_{S D}\right|^{2}+\left|h_{R_{1} D}\right|^{2}+\left|h_{R_{2} D}\right|^{2}+\left|h_{R_{3} D}\right|^{2}\right)$ is the power gain on the main diagonal and $\beta=\frac{2 \operatorname{Re}\left(h_{S D} h_{R_{3} D}^{*}-h_{R_{1} D} h_{R_{2} D}^{*}\right)}{h^{2}}$ is an additional interfering element at off-diagonal positions. The ML detector is now applied to $v$ for all pair of symbols $\left\{s_{1}, s_{4}\right\}$ and $\left\{s_{2}, s_{3}\right\}$ which minimizes the Euclidean distance $J$

$J_{\left(s_{1}, s_{4}\right)}=\frac{1}{1-\beta^{2}}\left(\left|v_{1}-h^{2}\left(s_{1}+\beta s_{4}\right)\right|^{2}+\left|v_{4}-h^{2}\left(s_{4}+\beta s_{1}\right)\right|^{2}\right)$

$-2 \beta \operatorname{Re}\left(\left[v_{1}-h^{2}\left(s_{1}+\beta s_{4}\right)\right]\left[v_{4}^{*}-h^{2}\left(s_{4}^{*}+\beta s_{1}^{*}\right)\right]\right)$

$J_{\left(s_{2}, s_{3}\right)}=\frac{1}{1-\beta^{2}}\left(\left|v_{2}-h^{2}\left(s_{2}+\beta s_{3}\right)\right|^{2}+\left|v_{3}-h^{2}\left(s_{3}+\beta s_{2}\right)\right|^{2}\right)$

$-2 \beta \operatorname{Re}\left(\left[v_{2}-h^{2}\left(s_{2}+\beta s_{3}\right)\right]\left[v_{3}^{*}-h^{2}\left(s_{3}^{*}+\beta s_{2}^{*}\right)\right]\right)$

The combiner now jointly recovers the estimates of the information signals $\widehat{S}_{1}$ and $\widehat{S}_{4}$, and $\widehat{S}_{2}$ and $\widehat{S}_{3}$ in pairs by combining (15) and (16) as follows:

$\widehat{s}_{1}+\widehat{s}_{4}=\left(r_{0} h_{S D_{1}}^{-1}+r_{i+3} h_{S D}^{*}+r_{i+4}^{*} h_{R_{1} D}+r_{i+5}^{*} h_{R_{2} D}+r_{i+6} h_{R_{3} D}^{*}\right)$

$+\left(r_{i+2} h_{S D_{1}}^{-1}+r_{i+3} h_{R_{3} D}^{*}-r_{i+4}^{*} h_{R_{2} D}-r_{i+5}^{*} h_{R_{1} D}+r_{i+6} h_{S D}^{*}\right)$

$=\left(s_{1}+s_{4}\right)\left(h_{2}+\beta_{2}\right)+Z_{1,4}$

$\widehat{s}_{2}+\widehat{s}_{3}=\left(r_{i} h_{S D_{1}}^{-1}+r_{i+3} h_{R_{1} D}^{*}-r_{i+4}^{*} h_{S D}+r_{i+5}^{*} h_{R_{3} D}-r_{i+6} h_{R_{2} D}^{*}\right)$

$+\left(r_{i+1} h_{S D_{1}}^{-1}+r_{i+3} h_{R_{2} D}^{*}+r_{i+4}^{*} h_{R_{3} D}-r_{i+5}^{*} h_{S D}-r_{i+6} h_{R_{1} D}^{*}\right)$

$=\left(s_{2}+s_{3}\right)\left(h_{2}-\beta_{2}\right)+Z_{2,3}$

$h_{2}=\left(1+\left|h_{S D}\right|^{2}+\left|h_{R_{1} D}\right|^{2}+\left|h_{R_{2} D}\right|^{2}+\left|h_{R_{3} D}\right|^{2}\right) \quad \beta_{2}=2 \operatorname{Re}\left(h_{S D} h_{R_{3} D}^{*}-h_{R_{1} D} h_{R_{2} D}^{*}\right)$

and $Z_{n}$ captures all the noise terms. We notice that switching $D$ to receiving mode at $t_{1}$ only slightly incurs an additional power gain on $h_{2}$ notably +1 compared to the DQSTBC proposed in [10], however, it does not increase the magnitude of the interfering element $\beta_{2}$, while still offering improved diversity performance.

\section{Simulation Results}

In this section, we show the simulated BER performance of SA-DOSTBC and SA-DQOSTBC and compare with the 
conventional DOSTBC and DQSTBC. All curves are plotted against the SNR at $D$ alone, and the BER is averaged over $10^{5}$ channel realizations (in the first part). First, the performance of the SA-DOSTBC using BPSK and 16QAM modulation schemes with Gray coding is considered. The results in Fig. 2 show that with BPSK the SA-DOSTBC achieves improved diversity performance of $2 \mathrm{~dB}$ at $\mathrm{BER}=10^{-2}$ for example in contrast to the performance of the conventional DOSTBC (see results in [4] for comparison). A similar phenomenon can be observed for the BER performance with 16QAM, again we can see that the proposed scheme is about $2 \mathrm{~dB}$ better than the conventional scheme. This implies that the theoretical result in (14) is consistent with the simulation result, thus we can conclude that the additional information signal received by switching $D$ to receiving mode at $t_{1}$ improves the BER performance of the network with negligible decoding complexity penalty.

Next, in Figure 3 we compare the SA-DQOSTBC with conventional DQOSTBC using BPSK and QPSK. Similarly from the results we can conclude that the SADQOSTBC outperforms the conventional DQSTBC, for example at $\mathrm{BER}=10^{-2}$, the proposed scheme with QPSK has about $1 \mathrm{~dB}$ improvement. It is also clear that the SA strategy offers more improvement for the orthogonal design in comparison to the quasi-orthogonal design, this is because the implementation of the SA strategy incurs much lower decoding complexity at $D$ for the orthogonal design.

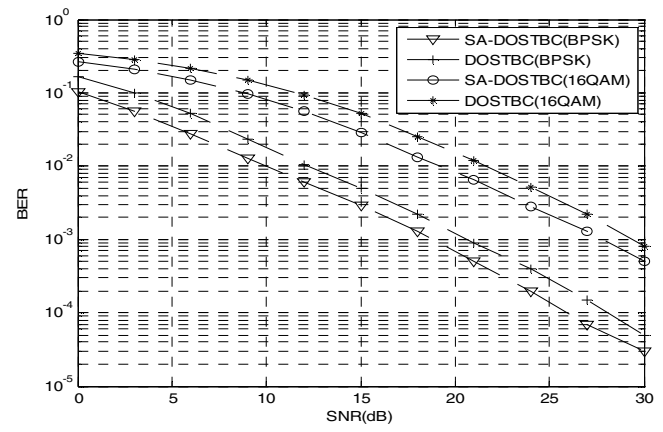

Fig.2. BER Performance of SA-DOSTBC

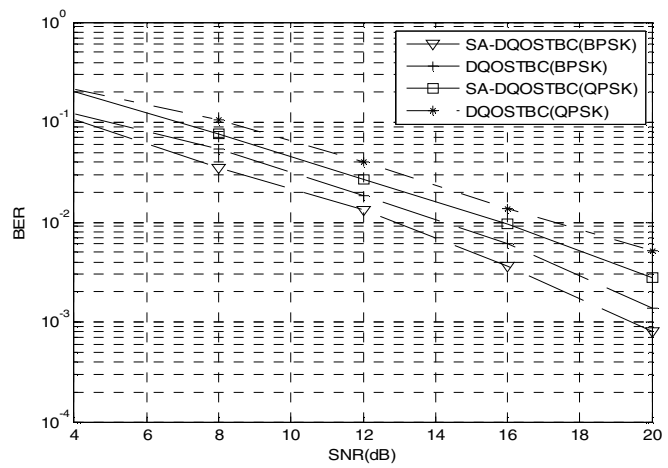

Fig.3. BER Performance of SA-DQOSTBC

\section{CONCLUSION}

The design of the SA strategy is considered for orthogonal and quasi-orthogonal distributed space-time codes using DF cooperative multi-hop networks. We identify that most works on DSTBC assume there is no direct $S-D$ link and thus ignore the benefits that can be extracted when the source is actively involved in cooperation. In this work, a two time-slot protocol is adopted and the SA design is implemented such that the destination takes advantage of the information signals transmitted from the source in both timeslots. This approach improves the diversity performance of the network at the expense of slightly increased complexity of the ML detector. The DSTBC design criteria is shown for generalized rate one orthogonal and quasi-orthogonal designs, we then provide an explanation on the modified structure of the ML detector and the improved diversity performance achieved by our proposed strategy.

\section{REFERENCES}

[1] M. N. Khormuji and E. G. Larsson, "Cooperative Transmission Based on Decode-And-Forward Relaying with Partial Repetition Coding", IEEE Transactions on Wireless Communications, Vol.8, Issue 4, April 2009

[2] D. Chen and J. N. Laneman, "Modulation and Demodulation for Cooperative Diversity in Wireless Systems," IEEE Transactions on Wireless Communications, Vol. 5, pp.1785-1794, July 2006

[3] P. A. Anghel and M. Kaveh, "On the Performance of Distributed Space-Time Coding Systems with One and Two Non-Regenerative Relays", IEEE Transactions on Wireless Communications, Vol. 5, No.3, March 2006

[4] K.G. Seddik, A. K. Sadek, A. S. Ibrahim, and K. J. R. Liu, "Design Criteria and Performance Analysis for Distributed Space-Time Coding", IEEE Transactions on Vehicular Technology, Vol.57, No. 4, July 2008

[5] J. N. Laneman, D. N. C. Tse, and G. W. Wornell, "Cooperative Diversity in Wireless Networks: Efficient Protocols and Outage Behaviour," IEEE Transactions on Information Theory, Vol.50, pp.3062-3080, December 2004

[6] B. Maham, A. Hjorungnesy and G. Abreuz, "Distributed GABBA Space-time Codes with Complex Signal Constellations", 5th IEEE Sensor Array and Multi-channel Signal Processing Workshop, pp.118 - 121, July 2008

[7] T. Cui, F. Gao, T. Ho and A. Nallanathan, "Distributed Space-Time Coding for Two-Way Wireless Relay Networks", IEEE Transactions on Signal Processing Vol. 57, Issue 2, pp. 658-671, February 2009

[8] G. S. Rajan and B. S. Rajan, "Distributed Space-Time Codes for Cooperative Networks with Partial CSI", IEEE Wireless Communications and Networking Conference, March 2007

[9] Z. Utkovski, G. Yammine and J. Lindner, "A Distributed Differential Space-time Coding Scheme for Two-way Wireless Relay Networks", IEEE International Symposium on Information Theory, pp.779 - 783, June 2009

[10] Y. Jing and H. Jafarkhani, "Using Orthogonal and Quasi-Orthogonal Designs in Wireless Relay Networks", IEEE Transactions on Information Theory, Vol. 53, No. 11, November 2007

[11] S. Alamouti, "A Simple Transmit Diversity Technique for Wireless Communications," IEEE Journal on Selected Areas in Communications, Vol.16, pp. 1451-1458, August 1998

[12] H. Jafarkhani, "A Quasi-orthogonal Space-time Block Code", IEEE Transactions on Communications, Vol. 49, pp.1-4, January 2001 